\title{
Dominance hierarchies among worker ants
}

\author{
from Robert M. May
}

THE pecking order of barnyard fowls and other birds, and the dominance hierarchies of hamadryas baboons and other primates (including academics), are widely known. One tends to think of such phenomena, however, as being found only among vertebrate species.

In a remarkable recent study, Cole (Science 212; 83, 1981) has demonstrated the existence of linear dominance hierarchies among the workers of the ant species, Leptothorax allardycei. These dominance hierarchies, moreover, have all the classic characteristics of their vertebrate analogues: routine displays of dominance, avoidance behaviour and even fighting.

Cole collected colonies of these neotropical myrmicine ants from their habitats among dead sawgrass stems in pine and palmetto scrub (in the Florida Keys) and housed them for observation in glass nests. Encounters between worker ants were then watched and the results tabulated in the conventional matrix form; these interaction matrices comprise totals of 200-400 interactions, over 10-20 hours of observation. In Cole's words, the agonistic encounters begin "with the dominant ant touching the submissive ant with its antennae. There is brief pause before the dominant either rapidly turns or lunges toward the subordinate. It then pummels the gaster, thorax, or head of the subordinate with its mandibles. The encounter may continue, with the dominant ant advancing onto the top of the

to its most basic simplicity. Or so the theorists claim.

Temperatures of $10^{30} \mathrm{~K}$ are, to say the least, inaccessible to present technology. There was one occasion, however, when matter could have been as hot as this: during the big bang. The creation of the Universe generated enormous quantities of heat, and it is generally supposed that the temperature of the primeval furnace plummetted from unlimited values at the outset to a mere $10^{10} \mathrm{~K}$ within one second. For about $10^{-35}$ seconds (which is brief even by physicists' standards), the temperature could have been high enough for the predicted ultra-simple phase of matter to have fleetingly existed.

Matter, and the forces that control it, are pictured as having frozen out of the featureless primeval soup in a succession of phase transactions, each with progressively more structure. The distinctive differences between leptons and quarks, and between electromagnetic, weak and strong nuclear forces, are regarded as a purely lowtemperature differentiation.

When water freezes to ice, it releases a great deal of heat - so-called latent heat - subordinate, sometimes climbing clear of the nest floor and standing on the subordinate. The typical response of the subordinate is to crouch and freeze, with the antennae drawn back to the sides of the head. It stays in this position until the dominant moves away." The dominance hierarchies thus established are almost perfectly linear, with less than 0.5 per cent of the interactions being 'reversals'. The highest ranking worker accounted for about 43 per cent of all dominance displays, and the second ranking for about 28 per cent; this is typical also of vertebrate dominance hierarchies (see, for example, Wilson Sociobiology, Harvard University Press, 1975), and is plausibly explained on the grounds that the higher the rank, the more worthwhile it is to expend effort in defending it.

Cole demonstrates two main advantages that accrue to the dominant workers. First, they are favoured in the exchange of liquid food. Although it is common for liquid food to be exchanged among workers in colonies of social insects, in L. allardycei this food transfer is always in one direction. High-ranking workers receive food from low-ranking ones, but do not reciprocate; Cole estimates the probability that chance alone could account for such apparently unidirectional transfer of food to be less than 0.001 .

Second, Cole showed that workers laid roughly 20 per cent of the colony's eggs. Robert M. May is Class of 1877 Professor of Zoology at Princeton University.

which is why water can seem reluctant to solidify at $0^{\circ} \mathrm{C}$. Similarly, in any phase transition, heat is either absorbed or expelled to help drive the transition itself, even without any change of temperature. Calculations suggest that when the quarklepton phase froze out of the primeval furnace, a large quantity of heat was also released.

This most exotic phase of matter has been studied by A.D. Linde of the Lebedev Physical Institute in Moscow. In a recent paper (Phys. Lett. 99B; 391, 1981), Linde argues that the quark-lepton freeze-out was an event of shattering proportions. What elevates the primeval latent heat release to the status of a 'bang' is the phenomenon of supercooling. It is well known that a very pure vapour can be carefully cooled to below its condensation temperature without liquid drops appearing. A slight disturbance will then lead to a catastrophic appearance of droplets. This principle was used in the socalled cloud chambers to detect the passage of subatomic particles.

Similarly in the primeval Universe, the quark-lepton phase transition did not
He did this by removing the queen for 3 days, and feeding the workers food in which Sudan black was mixed; Sudan black is fat soluble and not transferred in liquid food exchange, so that the queen is not contaminated with the stain when she is returned to the colony. As a result, the queen's eggs were the normal white colour, while those produced by workers (amounting, on average, to 21.8 per cent of the total) were stained bluc. The haplodiploid sexual system of these social insects means that the workers' eggs (which are unfertilized) produce males; the fertilized queen can produce males or females. Cole further demonstrated that the dominant workers had substantially greater ovarian development, and thus presumably accounted for most of the worker-produced males. In short, $L$. allardycei has effectively two reproductive castes: the queen, who produces all the females, and the highranking workers, who produce at least a significant fraction of the males.

The dominance hierarchies in $L$. allardycei constitute a most engaging curiosity. They also hold larger evolutionary implications. As Cole emphasizes: "this social organization ... has characteristics intermediate between those of the supposed nonsocial ancestors or ants and those of advanced eusocial species. Consideration of the social organization of this species must alter the conception of the reproductive options available to the worker caste of social insects." occur as soon as the temperature fell to the 'condensation' temperature. Because it depends on certain quantum 'tunnelling' processes that require a finite duration, the transition was inhibited until about $10^{-35} \mathrm{~s}$, by which time the temperature was down to a modest $10^{28} \mathrm{~K}$. When the transition finally occurred, in the words of Linde, it took on the features of a great explosion "shaking the whole Universe and changing abruptly its temperature".

The catastrophic release of energy suddenly reheated the cooling Universe and drastically altered the details of its evolution at a most sensitive epoch. Current theories suggest that it was at this stage that the Universe acquired its predominance of matter over antimatter, and that various enigmatic processes occurred which suppressed the production of magnetic monopoles. Linde calls this phase transition 'the grand bang' in honour of the so-called grand unified theories of fundamental forces that predict such a transition. No doubt its reverberations will disturb some of the tidy theories currently being formulated concerning the very early Universe. 